\title{
STRUCTURE-PRESERVING DISCRETIZATION IN THE FRAMEWORK OF A DISCRETE MODEL PROBLEM FOR LARGE-STRAIN THERMO-VISCOELASTICITY
}

\author{
Vanessa S. Valdes y Beck, Mark Schiebl and Peter Betsch \\ Institute of Mechanics, Karlsruhe Institute of Technology \\ Otto-Ammann-Platz 9, 76131 Karlsruhe, Germany \\ \{vanessa.beck, mark.schiebl, peter.betsch\}@kit.edu, http://www.ifm.kit.edu/
}

Key words: Coupled Thermo-Viscoelastic Problems, GENERIC Formalism, Structure-Preserving TimeStepping Schemes, Energy-Momentum-Entropy Integrators

\begin{abstract}
The design of structure-preserving integrators is dealt with in the framework of a discrete model problem for large-strain thermo-viscoelasticity. The evolution equations pertaining to the model problem are cast in the framework of GENERIC. The GENERIC formalism provides an ideal foundation for the design of energy-momentum-entropy (EME) consistent time-stepping schemes. An analogous approach can be applied to the full continuum model for large-strain thermo-viscoelasticity.
\end{abstract}

\section{INTRODUCTION}

A thermodynamic system's evolution equations can be cast in the General Equation for NonEquilibrium Reversible Irreversible Coupling (GENERIC) framework. GENERIC was developed by Grmela and Öttinger [1-3] and ensures the fulfilment of the two fundamental laws of thermodynamics.

The first GENERIC-based structure-preserving numerical method has been developed by Romero [4] in the context of a discrete model problem for thermo-elasticity. Similarly, in the present work we make use of a discrete model problem for thermo-viscoelasticity to outline the development of energymomentum-entropy (EME) consistent time-stepping schemes. Despite its simplicity, the model problem serves to convey main ideas of the design of EME methods for the full continuum model of large-strain thermo-viscoelasticity which has been recently addressed in [5].

While GENERIC-based numerical methods for continuum formulations of thermo-mechanically coupled solids have been dealt with in [6-11], various related discrete model problems have been the subject of previous work. For example, structure-preserving integrators have been developed in the context of a thermoelastic double pendulum [4, 12, 13], a thermo-viscoelastic element [14, 15], a thermo-viscoelastic double pendulum [8], and a thermo-elasto-plastic element [16].

These works proved an increased numerical stability and accuracy of structure-preserving timestepping schemes in comparison to standard integrators such as the the mid-point rule. However, their practicability strongly depends on the choice of the thermodynamic variable. Choosing the entropy or the internal energy as thermodynamic variable, requires the transformation of the constitutive relations, which can be cumbersome or even impossible. The choice of the temperature can instead lead to a complex structure of the resulting integrator. 
Mielke [17] exploited the advantageous transformation properties of GENERIC by introducing a generalized thermodynamic variable. This approach makes possible the free choice of the thermodynamic variable from among the entropy, temperature and internal energy. Recent developments of structurepreserving numerical methods in $[5,10,11,13,16]$ benefit from the possibility to use other thermodynamic variables than the entropy which has placed a severe restriction on earlier GENERIC-based schemes such as those in $[4,6,12]$.

An overview of the article is as follows. Section 2 introduces the thermo-viscoelastic model problem along with the state variables including the generalized thermodynamic variable. Section 3 summarizes the GENERIC-based formulation and points out its built-in thermodynamic correctness. This is the starting point for the formulation of a numerical time integration scheme based on the mid-point rule in Section 4. The mid-point integrator is evaluated with the choice of the entropy and temperature as thermodynamic state variable. Combining the GENERIC-based formulation with the discrete derivative due to Gonzalez [18] eventually yields the general formulation of EME schemes in Section 5. Numerical results shed light on the numerical properties of the present schemes.

\section{MODEL PROBLEM: THERMO-VISCOELASTIC DOUBLE PENDULUM}

The model problem at hand is depicted in Figure 1. The thermo-viscoelastic double pendulum consists of a thermo-spring and a generalized Maxwell element. The latter arranges a thermo-spring in parallel with a thermo-Maxwell element consisting of a spring in series with a damper. The thermospring attaches a point mass $m_{1}$ to a fixed point. The generalized Maxwell element connects $m_{1}$ to a

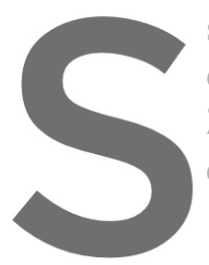
second point mass $m_{2}$. $q_{1}, q_{2} \in \mathbb{R}^{3}$. Each element $\lambda_{\alpha \cdot}^{0}$. The point masses' describe the viscoelastic
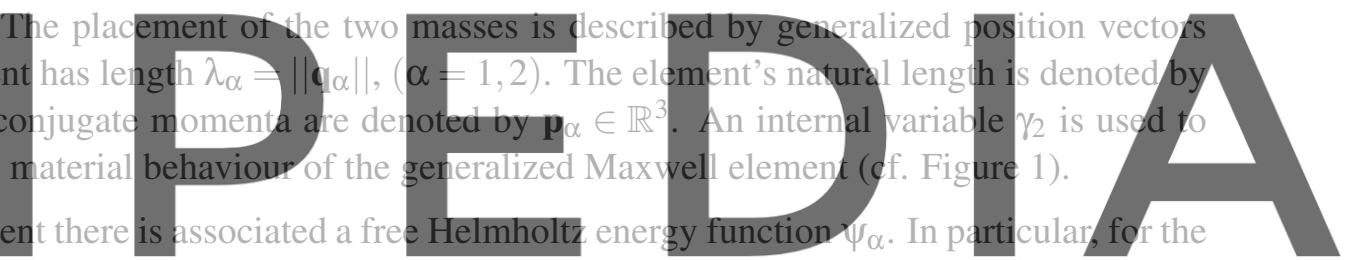

thermo- spring, $\psi_{1}=\psi_{1}\left(\lambda_{1}, \theta_{1}\right)$, while for the thermo-Maxwell element, $\psi_{2}=\psi_{2}\left(\lambda_{2}, \theta_{2}\right)$. Here, $\theta_{\alpha}$ is

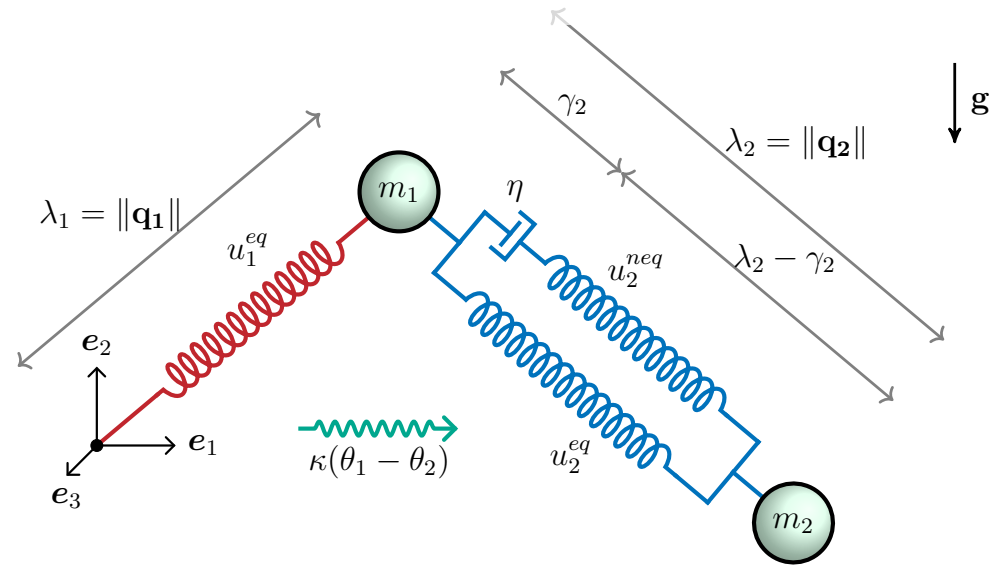

Figure 1: The thermo-viscoelastic double pendulum 
formation on relevant thermodynamic relationships we refer to [11, 17]. In particular, upon introduction of the generalized thermodynamic variable $\tau_{\alpha} \in\left\{s_{\alpha}, \theta_{\alpha}, u_{\alpha}\right\}$, the element's absolute temperature can be written in the form

$$
\theta_{\alpha}=\frac{\partial_{\tau_{\alpha}} u_{\alpha}}{\partial_{\tau_{\alpha}} s_{\alpha}}
$$

The model problem is complemented by (i) an evolution equation for the internal variable $\gamma_{2}$, and (ii) a law for the heat flux evoked by the temperature difference between the two thermo-elements.

The evolution in time of the present system in a time interval $I=[0, T]$ is described by the state vector $\mathbf{z}$, which takes the form

$$
\mathbf{z}=\left[\mathbf{q}_{1}^{\mathrm{T}}, \mathbf{q}_{2}^{\mathrm{T}}, \mathbf{p}_{1}^{\mathrm{T}}, \mathbf{p}_{2}^{\mathrm{T}}, \tau_{1}, \tau_{2}, \gamma_{2}\right]^{\mathrm{T}} .
$$

The state space $\mathcal{S}$ contains all admissible configurations and is given by

$$
\mathcal{S}=\left\{\mathbb{Z} \in\left(\mathbb{R}^{n d i m} \times \mathbb{R}^{n d i m} \times \mathbb{R}^{\text {ndim }} \times \mathbb{R}^{\text {ndim }} \times \mathbb{R} \times \mathbb{R} \times \mathbb{R}\right), \mathbb{q}_{1} \neq 0, \mathbb{q}_{2} \neq 0\right\}
$$

with $n$ dim $\in\{2,3\}$, being the spatial dimension.

\section{GENERIC-BASED FORMULATION}

The initial value problem governing the time-evolution of $\mathbf{z}: I \rightarrow S$ can now be formulated in the framework of GENERIC

building blocks. These cort and total entropy of the Poisson matrix and $\mathbf{M}$ the
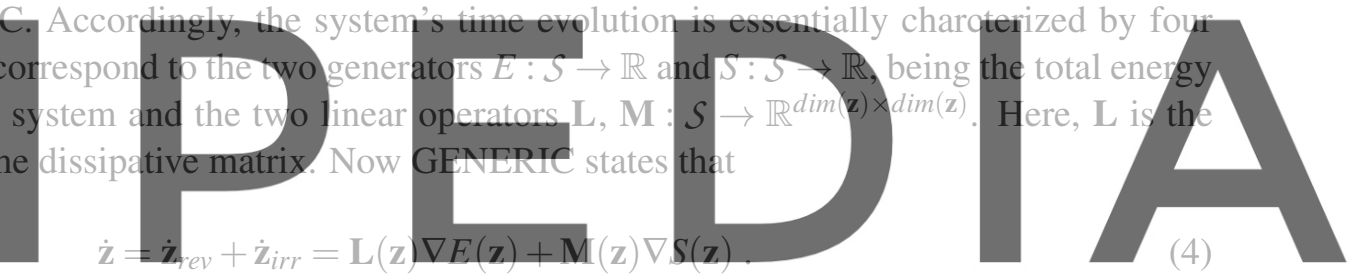

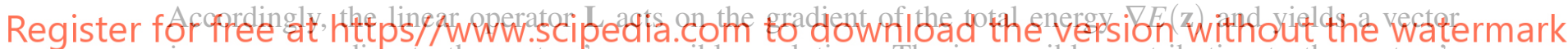

$\dot{\mathbf{Z}}_{\text {rev }}$ corresponding to the system's reversible evolution. The irreversible contribution to the system's evolution $\dot{\mathbf{Z}}_{i r r}$ is composed of the linear operator $\mathbf{M}$ and the gradient of the total entropy $\nabla S(\mathbb{Z})$. The initial conditions $\mathbf{z}(t=0)=\mathbf{z}^{0}$ complete the initial value problem.

For reasons of thermodynamic consistency, the following degeneracy or non-interaction-conditions have to be met

$$
\begin{aligned}
\mathbf{L} \nabla S & =\mathbf{0} \\
\mathbf{M} \nabla E & =\mathbf{0} .
\end{aligned}
$$

The Poisson and dissipative matrices have to comply with additional requirements. The first has to be skew symmetric, while the second has to be symmetric and positive semidefinite. Energy conservation as per first law of thermodynamics is achieved through the second degeneracy condition $(5)_{2}$ and the Poisson matrix's skew symmetry:

$$
\dot{E}(\mathbf{z})=\nabla E(\mathbf{z}) \cdot \dot{\mathbf{z}}=\nabla E(\mathbf{z}) \cdot \mathbf{L}(\mathbf{z}) \nabla E(\mathbf{z})+\nabla E(\mathbf{z}) \cdot \mathbf{M}(\mathbf{z}) \nabla S(\mathbf{z})=0 .
$$


Note that the present problem constitutes a closed system since (i) no external loads act, and (ii) the system is thermally insulated. Thus the total energy is indeed a conserved quantity as stated by (6). The second law of thermodynamics states entropy-production and is ensured by the first degeneracy condition $(5)_{1}$, together with the dissipative matrix's requirements of symmetry and positive semidefiniteness. It follows as

$$
\begin{aligned}
\dot{S}(\mathbf{z}) & =\nabla S(\mathbf{z}) \cdot \dot{\mathbf{z}}=\nabla S(\mathbf{z}) \cdot \mathbf{L}(\mathbf{z}) \nabla E(\mathbf{z})+\nabla S(\mathbf{z}) \cdot \mathbf{M}(\mathbf{z}) \nabla S(\mathbf{z}) \\
& =\nabla S(\mathbf{z}) \cdot \mathbf{M}(\mathbf{z}) \nabla S(\mathbf{z}) \geq 0 .
\end{aligned}
$$

It can be shown that the Poisson matrix $\mathbf{L}$ and dissipative matrix $\mathbf{M}$ are given by

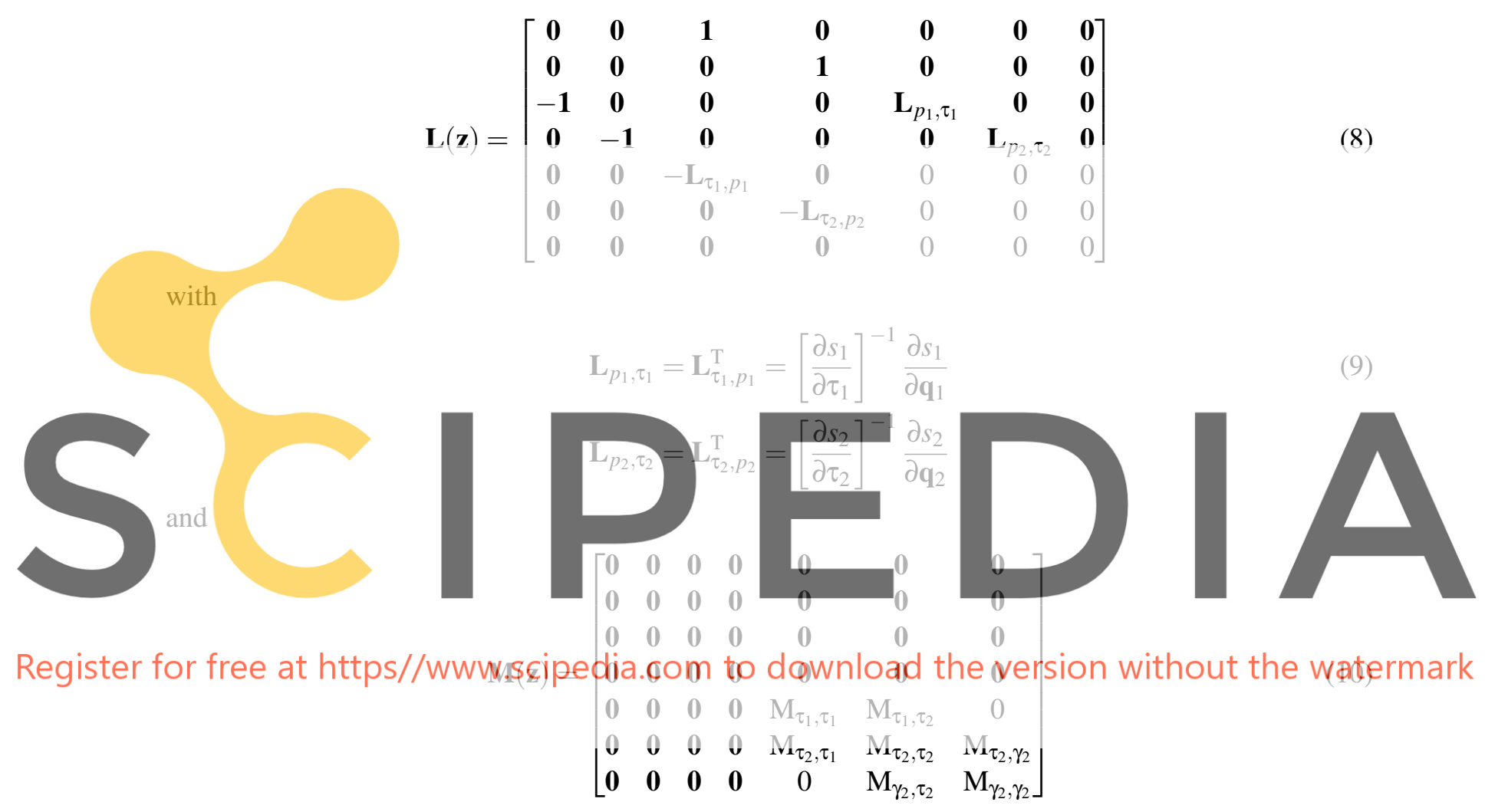

with

$$
\begin{aligned}
\mathrm{M}_{\tau_{1}, \tau_{1}} & =\left[\frac{\partial s_{1}}{\partial \tau_{1}}\right]^{-2} \kappa \frac{\theta_{2}}{\theta_{1}} \\
\mathrm{M}_{\tau_{1}, \tau_{2}} & =\mathrm{M}_{\tau_{2}, \tau_{1}}=-\kappa\left[\frac{\partial s_{1}}{\partial \tau_{1}}\right]^{-1}\left[\frac{\partial s_{2}}{\partial \tau_{2}}\right]^{-1} \\
\mathrm{M}_{\tau_{2}, \tau_{2}} & =\left[\frac{\partial s_{2}}{\partial \tau_{2}}\right]^{-2} \eta^{-1} \theta_{2}{ }^{-1}\left[\frac{\partial u_{2}}{\partial \gamma_{2}}\right]^{2}+\left[\frac{\partial s_{2}}{\partial \tau_{2}}\right]^{-2} \kappa \frac{\theta_{1}}{\theta_{2}} \\
\mathrm{M}_{\tau_{2}, \gamma_{2}} & =\mathrm{M}_{\gamma_{2}, \tau_{2}}=-\left[\frac{\partial s_{2}}{\partial \tau_{2}}\right]^{-1} \eta^{-1} \frac{\partial u_{2}}{\partial \gamma_{2}} \\
\mathrm{M}_{\gamma_{2}, \gamma_{2}} & =\eta^{-1} \theta_{2} .
\end{aligned}
$$


The total energy for the system at hand consists of the kinetic energy $T$, in addition to the total internal energy $U=u_{1}+u_{2}$ and the potential $V$ due to gravity:

$$
E(\mathbf{z})=T\left(\mathbf{p}_{1}, \mathbf{p}_{2}\right)+u_{1}\left(\mathbf{q}_{1}, \tau_{1}\right)+u_{2}\left(\mathbf{q}_{2}, \tau_{2}, \gamma_{2}\right)+V\left(\mathbf{q}_{1}, \mathbf{q}_{2}\right) .
$$

The total entropy corresponds to

$$
S(\mathbf{z})=s_{1}\left(\mathbf{q}_{1}, \tau_{1}\right)+s_{2}\left(\mathbf{q}_{2}, \tau_{2}, \gamma_{2}\right) .
$$

The gradients of the total energy (12) and the total entropy (13) complete the evolution equations (4)

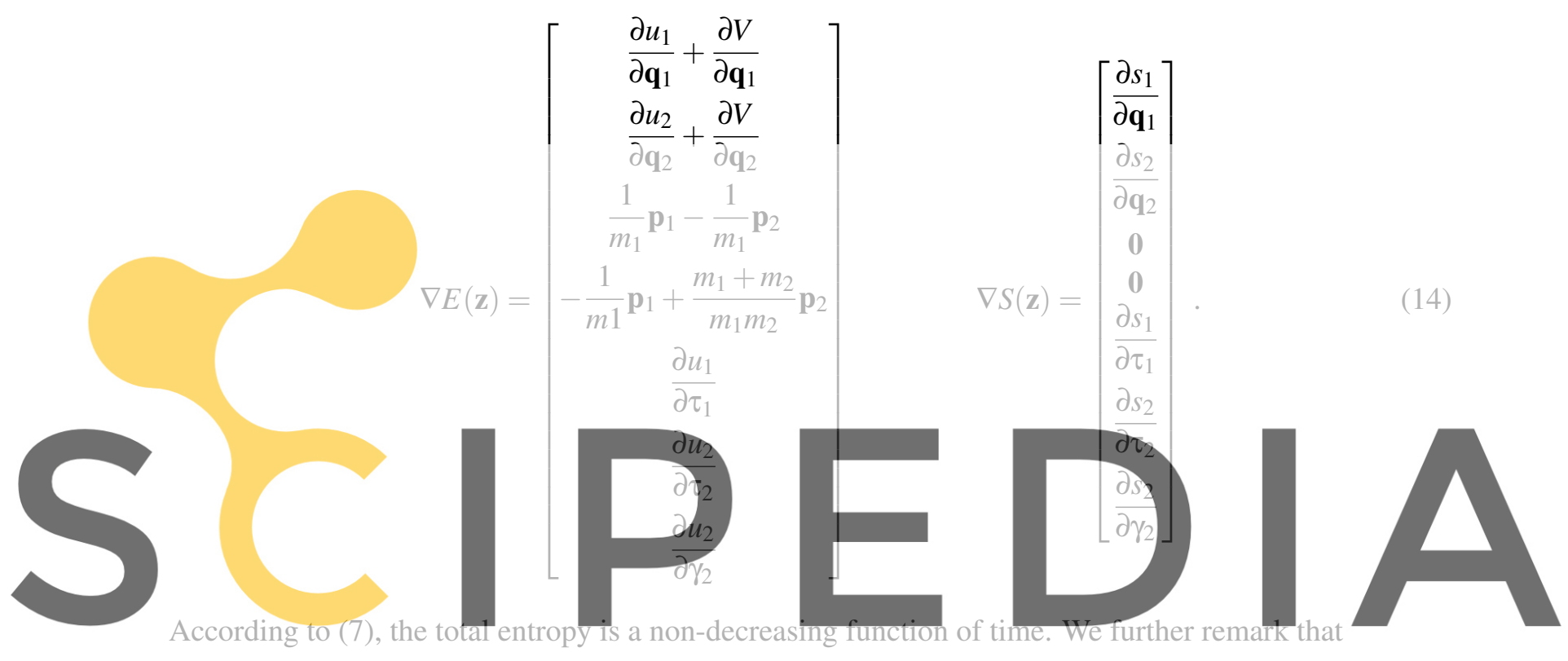

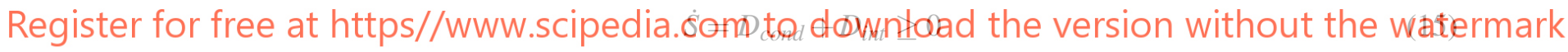

where the dissipation due to heat conduction is given by

$$
D_{\text {cond }}=\kappa \frac{\left[\theta_{2}-\theta_{1}\right]^{2}}{\theta_{1} \theta_{2}} \geq 0
$$

while

$$
D_{\text {int }}=\theta_{2}^{-1} \frac{\partial u_{2}}{\partial \gamma_{2}} \eta^{-1} \frac{\partial u_{2}}{\partial \gamma_{2}} \geq 0
$$

is the dissipation due to inelastic (viscous) deformation.

\section{NUMERICAL INTEGRATION APPLYING THE MID-POINT RULE}

This section deals with the numerical integration of the evolution equation (4) by means of the midpoint rule. The time interval $I=[0, T]$ is discretized into finite time intervals $I_{n}=\left(t_{n-1}, t_{n}\right)$ with time 
step size $\Delta t=t_{n+1}-t_{n}$. The functional values at time $t_{n}$ are denoted by $(\bullet)_{n}$ and at time $t_{n+1}$ by $(\bullet)_{n+1}$. Application of the mid-point rule yields

$$
\frac{\mathbf{z}_{n+1}-\mathbf{z}_{n}}{\Delta t}=\mathrm{L}\left(\mathbf{z}_{n+\frac{1}{2}}\right) \nabla \mathrm{E}\left(\mathbf{z}_{n+\frac{1}{2}}\right)+\mathrm{M}\left(\mathbf{z}_{n+\frac{1}{2}}\right) \nabla \mathrm{S}\left(\mathbf{z}_{n+\frac{1}{2}}\right)
$$

It can be shown that the mid-point rule conserves symmetries and correctly displays the total entropy, when using the entropy as thermodynamic variable (see $[5,11]$ for further details). Thus in the following this integrator is referred to as (ME) (Momentum-Entropy). The selection of the internal energy as thermodynamic variable yields a correct energy behavior, therefore in this case the integrator is denoted as $(\mathrm{EM})_{u}$ (Energy-Momentum). However, the temperature as thermodynamic variable is not able to fulfil any of the two laws of thermodynamics, therefore it results in the $(\mathrm{M})_{\theta}$ integrator. For a detailed explanation we refer to [19].

In the numerical simulations the mid-point integrator is evaluated for the choice of the entropy and temperature as thermodynamic variable (i.e. the $(\mathrm{ME})_{s}$ and $(\mathrm{M})_{\theta}$ integrator), respectively. The free Helmholtz energy function is chosen similarly to [4] with

$$
\bar{\psi}=\bar{\psi}_{1}^{e q}\left(\lambda_{1}, \theta_{1}\right)+\bar{\psi}_{2}^{e q}\left(\lambda_{2}, \theta_{2}\right)+\bar{\psi}_{2}^{n e q}\left(\lambda_{2}, \theta_{2}, \gamma_{2}\right) .
$$

It is composed of the spring's free energy function

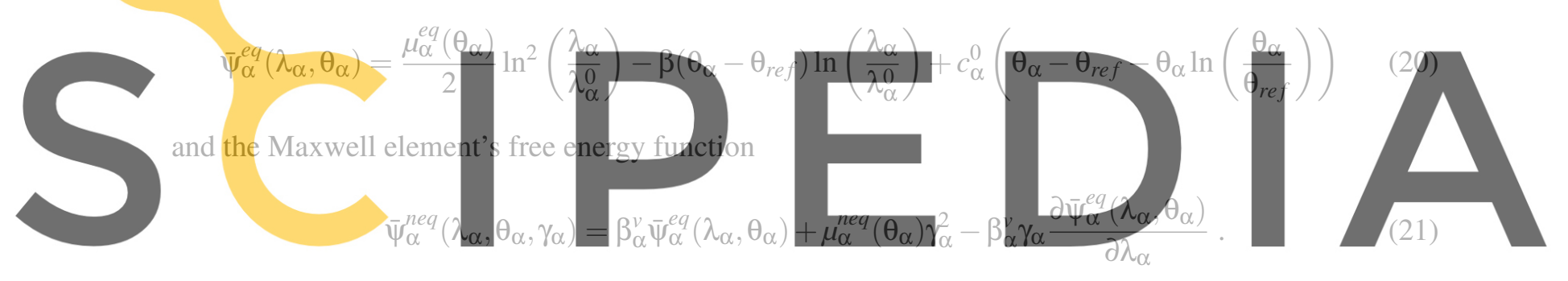

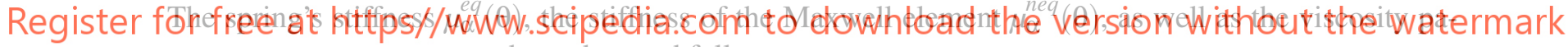
rameter $\eta$ are temperature-dependent and follow as

$$
\begin{array}{lll}
\mu_{\alpha}^{e q}(\theta)=\mu_{\alpha, c s t}^{e q}-\mu_{\alpha, \theta}^{e q}\left(\theta-\theta_{\text {ref }}\right) & & \mu_{\alpha, c s t}^{e q}, \mu_{\alpha, \theta}^{e q}>0 \\
\mu_{\alpha}^{e q}(\theta)=\mu_{\alpha, c s t}^{\text {neq }}-\mu_{\alpha, \theta}^{\text {neq }}\left(\theta-\theta_{\text {ref }}\right) & \text { with } & \mu_{\alpha, c s t}^{e q}, \mu_{\alpha, \theta}^{\text {neq }}>0 \\
\eta=\eta_{c s t} \exp \left[\eta_{\theta}\left(\frac{1}{\theta}-\frac{1}{\theta_{\text {ref }}}\right)\right] & & \eta_{c s t}, \eta_{\theta}>0 .
\end{array}
$$

Here, $c_{\alpha}^{0}, \theta_{\text {ref }}, \beta$ and $\beta^{v}$ denote the specific heat capacity, reference temperature and coupling parameters. The employed simulation parameters are summarized in Table 1 . Within the time interval of $I=[0,20]$ $\mathrm{s}$, three different constant time step sizes of $\Delta t \in\{0.005,0.12,0.18\} \mathrm{s}$ are applied. The initial conditions are chosen as:

$$
\theta_{1}^{0}=280 \mathrm{~K}, \quad \theta_{2}^{0}=350 \mathrm{~K}, \quad \mathbf{q}_{1}^{0}=\left[\begin{array}{l}
1 \\
0 \\
0
\end{array}\right] \mathrm{m}, \quad \mathbf{q}_{2}^{0}=\left[\begin{array}{l}
3 \\
0 \\
0
\end{array}\right] \mathrm{m}, \quad \mathbf{p}_{1}^{0}=\left[\begin{array}{l}
0 \\
0 \\
0
\end{array}\right] \mathrm{Ns}, \quad \mathbf{q}_{2}^{0}=\left[\begin{array}{c}
0 \\
0 \\
-1
\end{array}\right] \mathrm{Ns}, \quad \gamma_{2}^{0}=0 \mathrm{~m} .
$$




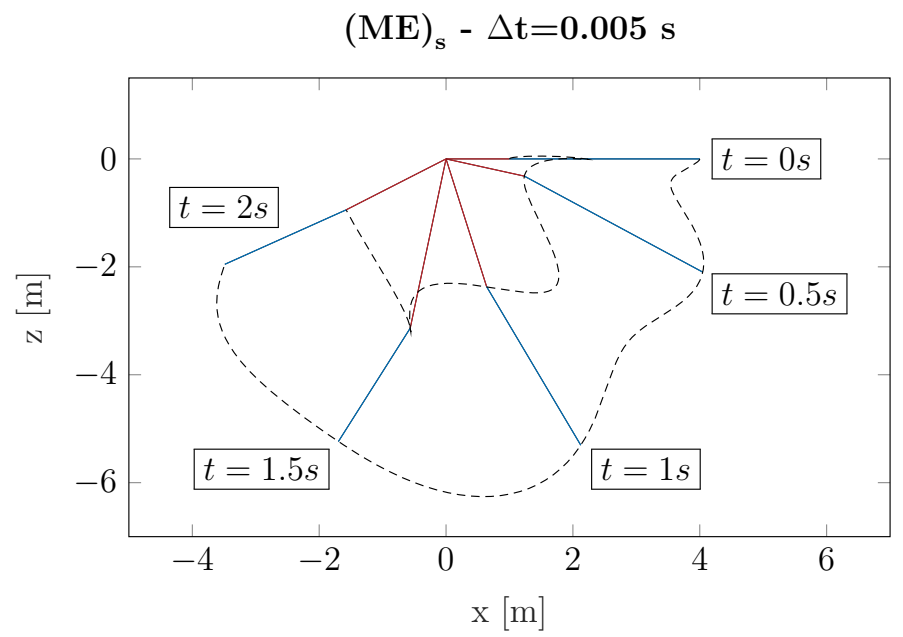

Figure 2: Motion of the double pendulum in the time interval $I=[0,2] \mathrm{s}$

Table 1: Numerical data for the thermo-viscoelastic double pendulum
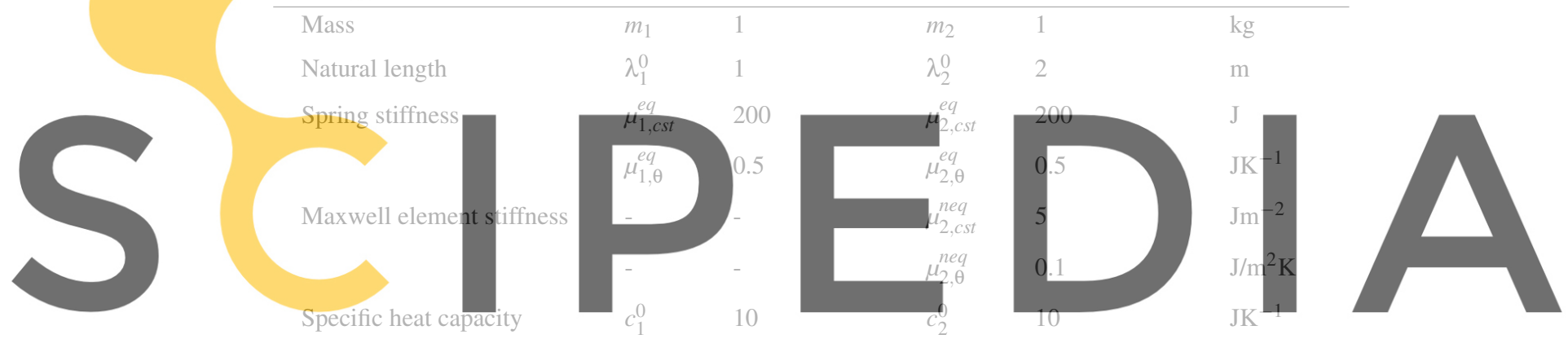

Register for free at hiscity

Thermal conductivity

Reference temperature

Coupling parameter

Gravitational vector

Newton tolerance k $\quad 1$

$\theta_{\text {ref }} \quad 300$

$\beta^{v} \quad 0.5$

g $\quad[0,0,-9.81]^{\mathrm{T}}$

$10 \mathrm{e}-8$
K

$\mathrm{JK}^{-1}$

$\mathrm{m}^{-2}$

$\mathrm{JK}^{-1} \mathrm{~S}^{-1}$

$\mathrm{ms}^{-2}$

The trajectories of the double pendulum's point masses in a time interval $I=[0,2] \mathrm{s}$ are displayed in Figure 2. Figure 3 (top) illustrates the system's total energy. The solution for the small time step size $\Delta t=0.005 \mathrm{~s}$ is nearly identical for both integrators and approximately energy conserving. Applying the intermediate time step size $\Delta t=0.12 \mathrm{~s}$ results in small fluctuations. The large time step size $\Delta t=0.18$ $\mathrm{s}$ displays a sharp increase in the course of the total energy, which points out a possible loss of stability. This results in an 'energy blow-up' when applying larger time step sizes. 


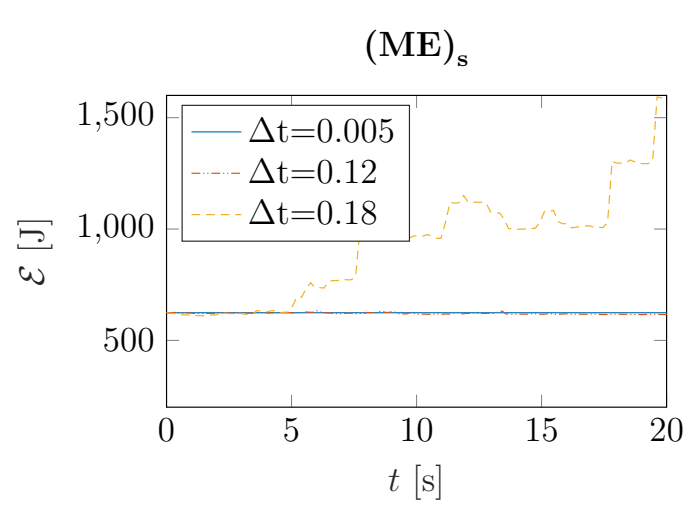

$(\mathrm{ME})_{\mathrm{s}}$
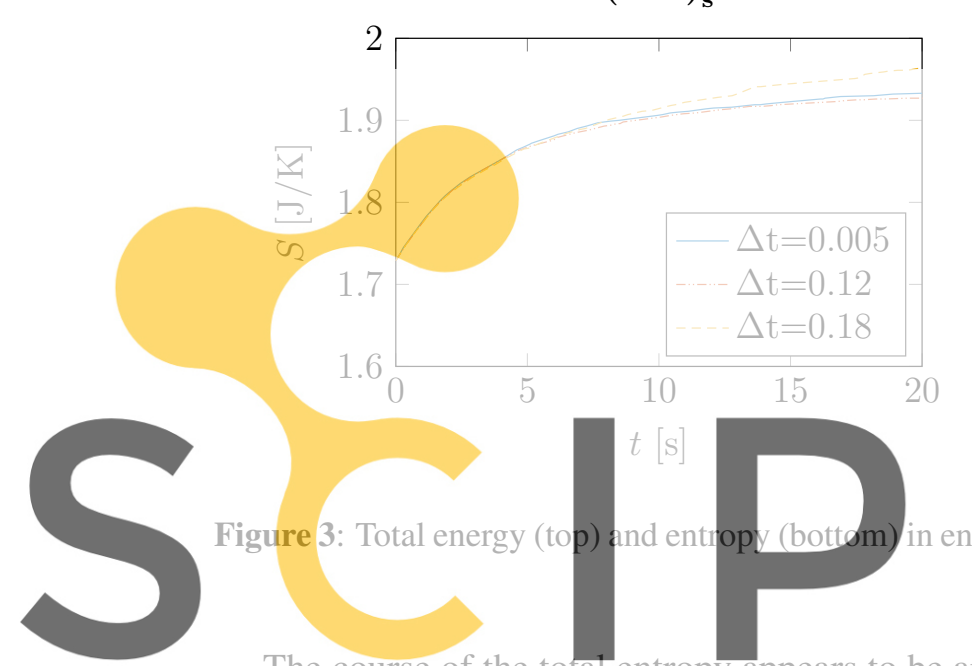

Figure 3: Total energy (top)

The course of the total entropy appears to be ap

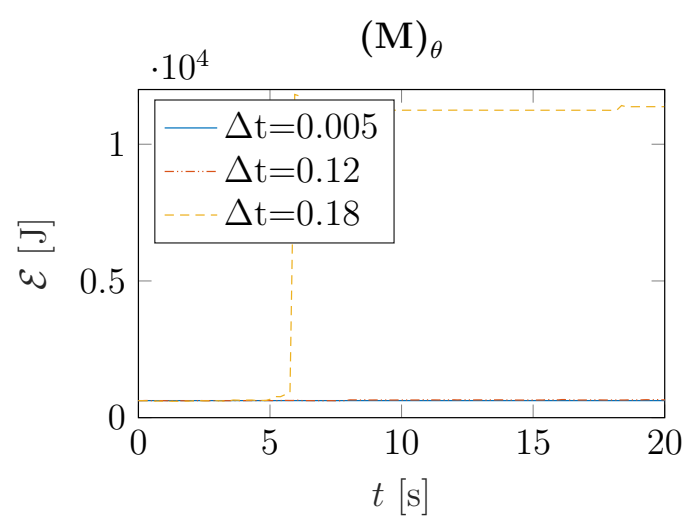

$(\mathrm{M})_{\theta}$

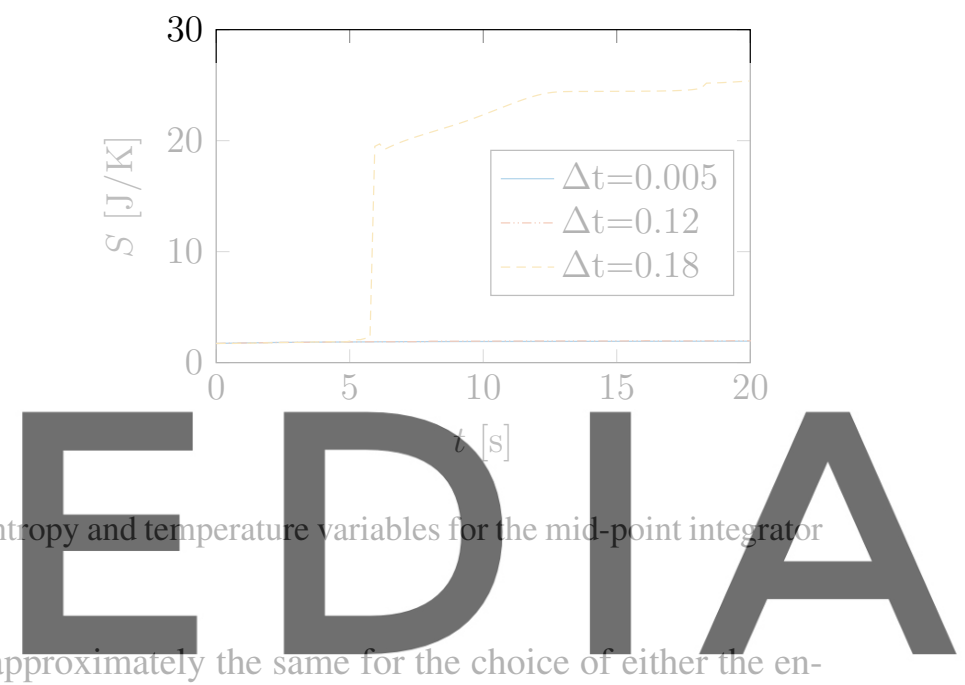

tropy or the temperature as thermodynamic variable when using small time step sizes (Figure 3 bottom).

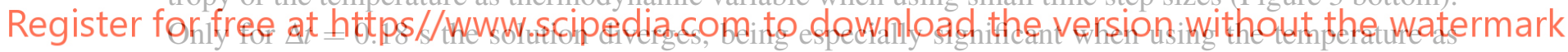

state variable. Although Figure 3 (bottom) seems to comply with the second law of thermodynamics, the

incremental change of the entropy shows that only the (MIE)s integrator is able to fulfil the second law of thermodynamics. The choice of the temperature as thermodynamic variable leads to a decrease of the total entropy and therefore violates the second law.

The lack of control over the energy and entropy is clearly evident when examining the course of the Lyapunov function (Figure 4) as criterion for numerical stability. The Lyapunov function is calculated by

$$
V=E-\theta_{r e f} S .
$$

Thus, numerical stability is characterized by a steadily decreasing Lyapunov function. This is clearly violated for large time steps. 

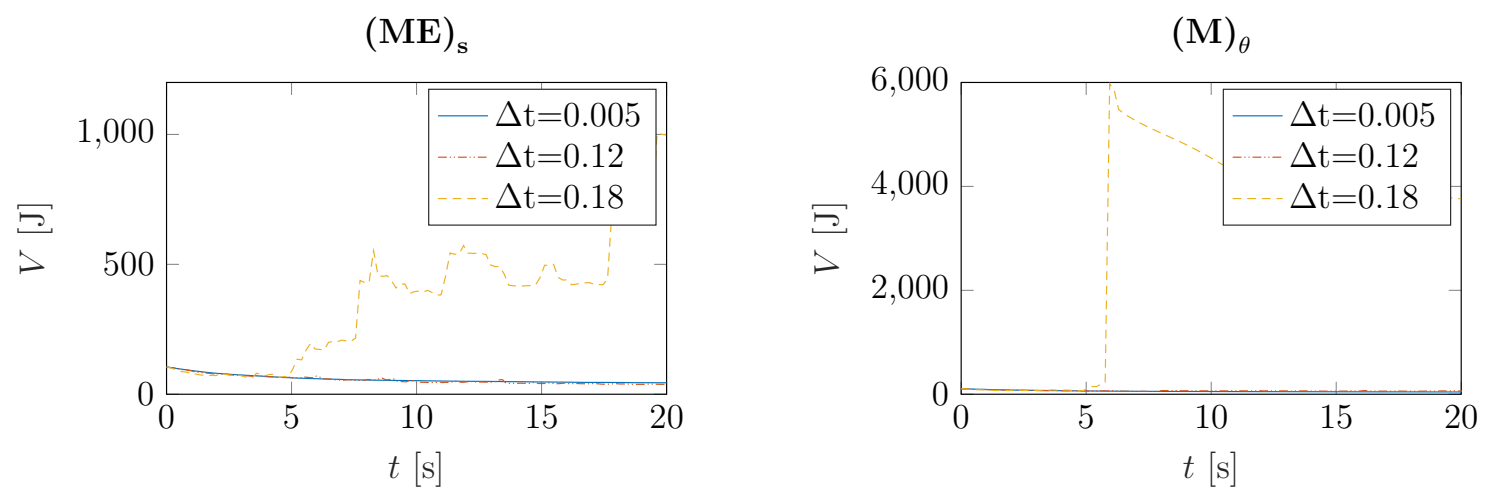

Figure 4: Lyapunov function in entropy and temperature variables for the mid-point integrator

The evolution of the length, temperature and internal variable display nonphysical behaviour for large time step sizes. This can be expected, judging from the violation of the first and second law of thermodynamics.

5 STRUCTURE-PRESERVING NUMERICAL INTEGRATION

Energy-momentum-entropy time-stepping schemes are thermodynamic consistent and symmetry pre-

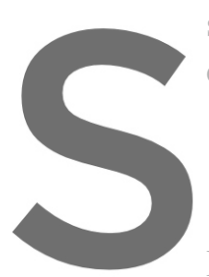
serving. Applying the discrete gradient operator D of GENERIC (4) can be wh

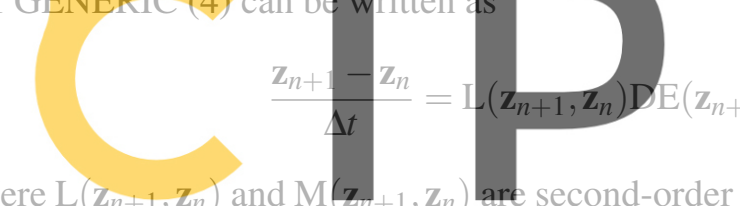

Here $\mathrm{L}\left(\mathbf{z}_{n+1}, \mathbf{z}_{n}\right)$ and $\mathrm{M}\left(\mathbf{z}_{n+1}, \mathbf{Z}_{n}\right)$ are second-order
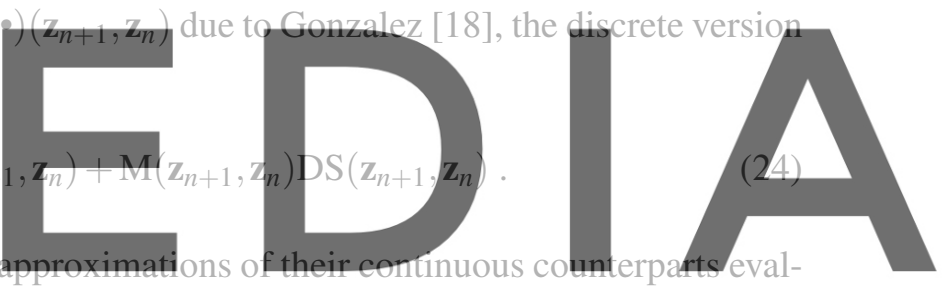

uated by employing the discrete gradient operator. The discrete gradient of a function $f(\mathbf{z})$ can be viewed

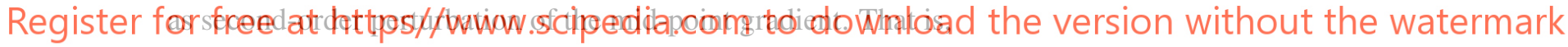

$$
\operatorname{Df}\left(\mathbf{z}_{n+1}, \mathbb{Z}_{n}\right)=\nabla f\left(\mathbb{z}_{n+\frac{1}{2}}\right)+\mathcal{O}\left(\Delta t^{2}\right)
$$

More details about discrete gradients and their use in the design of GENERIC-based EME schemes can be found in $[5,10]$.

The EME time-stepping scheme is evaluated for the choice of the entropy and the temperature as thermodynamic variable, leading to corresponding integrators denoted by $(\mathrm{EME})_{s}$ and $(\mathrm{EME})_{\theta}$, respectively. The numerical simulations are displayed with the large time step size $\Delta t=0.18 \mathrm{~s}$, for which the midpoint rule yielded unphysical results.

As expected, the EME schemes respect the two fundamental laws of thermodynamics, independent of the time step size. That is, the total energy is conserved (up to numerical round-off) and the total entropy is steadily increasing with time. This physically correct behavior of the EME schemes is accompanied by enhanced numerical stability as can be observed from the course of the Lyapunov function shown in Figure 5. 

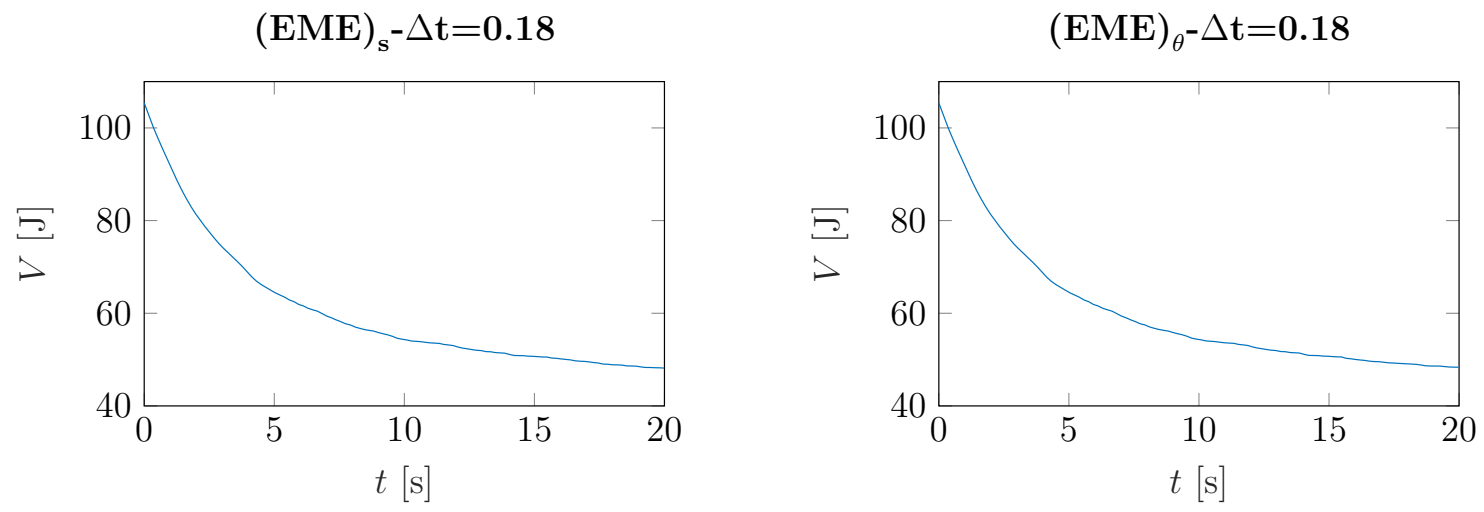

Figure 5: Lyapunov function in entropy and temperature variables for the EME integrator
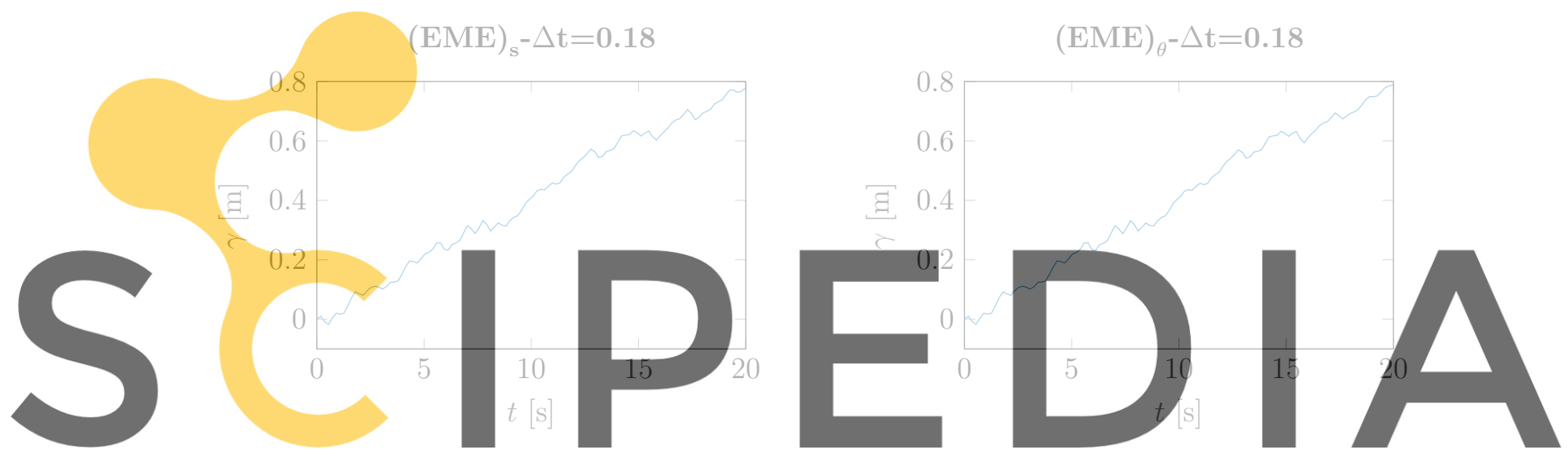

Figure 6: Internal variable (bottom) in entropy and temperature variables for the EME integrator Register for free at https//www.scipedia.com to download the version without the watermark

The time-evolution of the internal variable (Figure 6) and both length and temperature variables (Figure 7) is practically identical for both EME integrators under consideration. 
$(\mathrm{EME})_{\mathrm{s}}-\Delta \mathrm{t}=0.18$

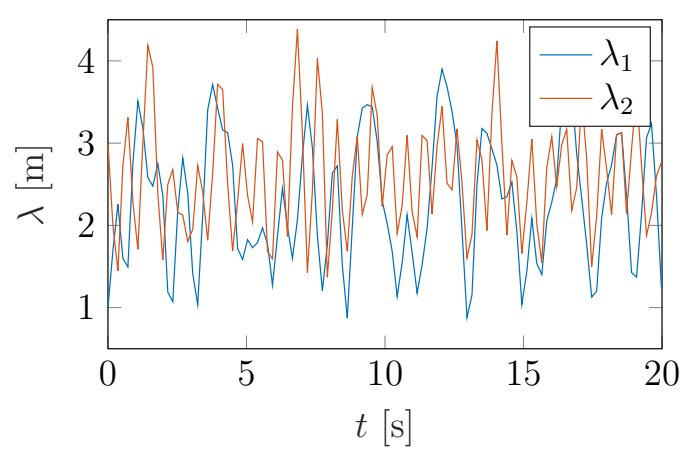

$(\mathrm{EME})_{\mathrm{s}}-\Delta \mathrm{t}=0.18$

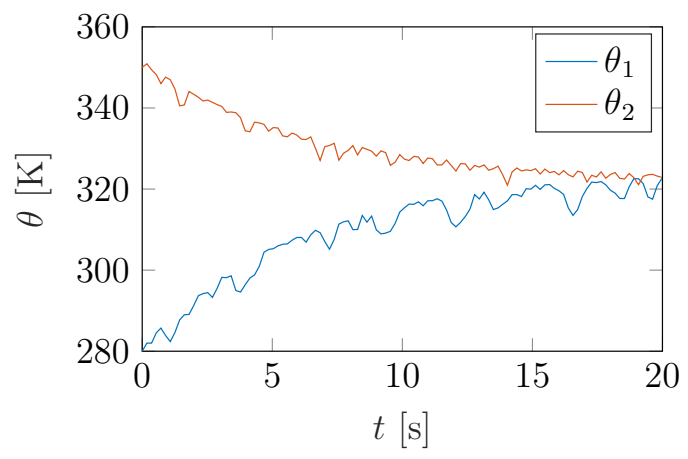

$(\mathrm{EME})_{\theta}-\Delta \mathrm{t}=\mathbf{0 . 1 8}$

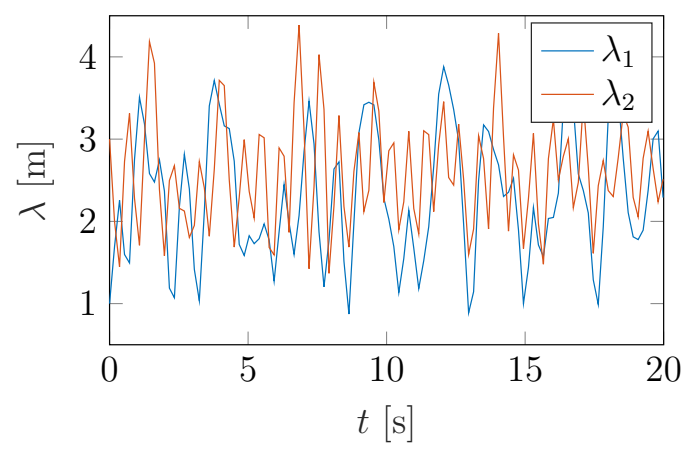

$(\mathrm{EME})_{\theta}-\Delta \mathrm{t}=\mathbf{0 . 1 8}$

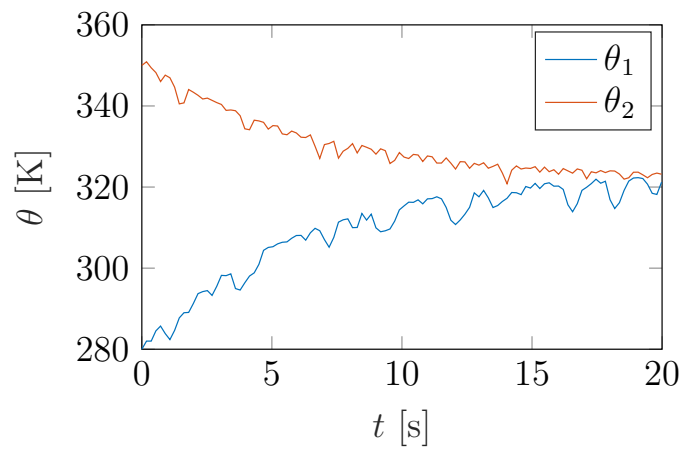

Figure 7: Element length and temperature in entropy and temperature variables for the EME integrator

As seen, the EME integrators are structure-preserving, as well as significantly more precise and numerically stable than the mid-point rule. Either in entropy or temperature variables, the EME integrators yield practically identical results.

\section{Acknowledgement}

Support for this research was provided by the Deutsche Forschungsgemeinschaft (DFG) under Grant BE 2285/13-1. This support is gratefully acknowledged.

\section{REFERENCES}

1. Grmela, M. \& Öttinger, H. C. Dynamics and thermodynamics of complex fluids - I. Development of a general formalism. PHYSICAL REVIEW E 56, 6620-6632 (1997).

2. Öttinger, H. C. \& Grmela, M. Dynamics and thermodynamics of complex fluids - II. Illustrations of a general formalism. PHYSICAL REVIEW E 56, 6633-6655 (1997).

3. Öttinger, H. C. Beyond equilibrium thermodynamics. (John Wiley \& Sons Inc., 2005).

4. Romero, I. Thermodynamically consistent time-stepping algorithms for nonlinear thermomechanical systems. Int. J. Numer. Meth. Engng 79, 706-732 (2009).

5. Schiebl, M. \& Betsch, P. Structure-preserving space-time discretization of large-strain thermoviscoelasticity in the framework of GENERIC. (Submitted for publication, December 15, 2020.). 
6. Romero, I. Algorithms for coupled problems that preserve symmetries and the laws of thermodynamics Part I. Monolithic integrators and their application to finite strain thermoelasticity. Comput. Methods. Appl. Mech. Eng. 199, 1841-1858 (2010).

7. Romero, I. Algorithms for coupled problems that preserve symmetries and the laws of thermodynamics. Part II. Fractional step methods. Comput. Methods. Appl. Mech. Eng. 199, 2235-2248 (2010).

8. Krüger, M. Energie-Entropie-konsistente Zeitintegratoren für die nichtlineare Thermoviskoelastodynamik. $\mathrm{PhD}$ thesis (Universität Siegen, 2013).

9. Krüger, M., Groß, M. \& Betsch, P. An energy-entropy-consistent time stepping scheme for nonlinear thermo-viscoelastic continua. ZAMM 96, 141-178 (2016).

10. Betsch, P. \& Schiebl, M. Energy-Momentum-Entropy consistent numerical methods for large strain thermoelasticity relying on the GENERIC formalism. Int. J. Numer. Meth. Engng 119, 1216-1244 (2019).

11. Betsch, P. \& Schiebl, M. GENERIC-based formulation and discretization of initial boundary value problems for finite strain thermoelasticity. Computational Mechanics 65, 503-531 (2020).

12. Krüger, M., Groß, M. \& Betsch, P. A comparison of structure-preserving integrators for discrete thermoelastic systems. Comput. Mech. 47, 701-721 (2011).

13. Conde Martín, S., Betsch, P. \& García Orden, J. C. A temperature-based thermodynamically consistent integration scheme for discrete thermo-elastodynamics. Commun Nonlinear Sci Numer Simulat 32, 63-80 (2016).

14. García Orden, J. C. \& Romero, I. Energy-Entropy-Momentum integration of discrete thermo-viscoelastic dynamics. Eur. J. Mech. A/Solids 32, 76-87 (2011).

15. Conde Martín, S. \& García Orden, J. C. On Energy-Entropy-Momentum integration methods for discrete thermo-visco-elastodynamics. Computers and Structures 181, 3-20 (2017).

16. Portillo, D., García Orden, J. C. \& Romero, I. Energy-entropy-momentum integration schemes for general discrete non-smooth dissipative problems in thermomechanics. Int. J. Numer. Meth. Engng 112, 776-802 (2017).

17. Mielke, A. Formulation of thermoelastic dissipative material behavior using GENERIC. Continuum Mech. Thermodyn. 23, 233-256 (2011).

18. Gonzalez, O. Design and analysis of conserving integrators for nonlinear Hamiltonian systems with symmetry. $\mathrm{PhD}$ thesis (Stanford University, 1996).

19. Valdes y Beck, V. S. Thermodynamically consistent integration of coupled thermoviscoelastic discrete systems. MA thesis (Karlsruhe Institute of Technology, 2020). 